\title{
EVALUATION OF THE POTENTIAL ACCURACY OF THE PULSED RADIO SIGNALS SOURCE COORDINATE DETERMINATION BY THE POSITIONING SYSTEM USING A SINGLE UAV-SENSOR
}

\author{
Baldytchev M.T. ${ }^{1}$, Laptev I.V. ${ }^{1}$, Chebotar I.V. ${ }^{1}$, Gaychuk Y.N. ${ }^{1}$, Pivkin I.G. ${ }^{1}$, \\ Timoshenko A.V. ${ }^{2}$, Galtseva O.V. ${ }^{3}$ \\ ${ }^{1}$ Cherepovets Higher Military Engineering School of Radio Electronics, Cherepovetc, Russia \\ 2OJSC Academician A.L. Mints Radiotechnical Institute, Moscow, Russia \\ 3National Research Tomsk Polytechnic University, Tomsk, Russia, rabota2013tpu@mail.ru
}

\begin{abstract}
An approach to determining the coordinates of pulsed radio signals sources by the positioning system in the application of a single unmanned aerial vehicles sensor is shown in this article. The range-difference location method allows to determinate an informative coordinate parameter without retransmission and internal synchronization of receiving points. The result is achieved by improving the well-known scientific and methodological apparatus with considering some features of the operation mode of pulsed radio signals sources. Presenting results of the simulation allow to estimate the influence of main input factors on the potential accuracy of the pulsed radio signal source coordinate determination.
\end{abstract}

Keywords: coordinate-informative parameter, unmanned aerial vehicles sensor, pulsed radio signals sources, the range-difference location method.

\section{Introduction}

Nowadays, pulsed radio signal source coordinate determination by passive means of monitoring is realized by the goniometrical method [1-8] or the range-difference location method. Methods implementation requires two or three receiving points equipped with radio monitoring and communication equipment, which allows have data link. The distance between them must be from one to ten kilometers. Achieving the power availability of pulsed radio signals sources (PRSS), receiving centers are accommodated on unmanned aerial vehicles (UAV). These vehicles have been widely spread nowadays. However, the use of UAVs as a platform for radio monitoring tools for solving the coordinates determination problems of radio emission sources, leads to a number of problems. The main of which are connected with weight and size limitations of the UAV payload, which do not allow to place effective direction finding antenna systems and repeater; instability of UAV spatial orientation, which leads to a sharp increase in errors of direction finding. Along with this, it is known that the smallest error in determining the location (of the existing passive methods of determining the coordinates) provides the range-difference location method (RDLM) [9-15].

The features of the range-difference location method include the simplicity of the antennafeeder system, as well as the invariance to the deviation of the angles of pitch, roll and yaw of the UAV. However, the implementation of classical range-difference location method requires the use of three or more UAVs equipped with radio monitoring tools and communication channels with high bandwidth, necessary for relaying the implementation of the received signal for correlation processing and measurement of the coordinate-informative parameter (CIP).

These features significantly complicate the structure and cost of used equipment, as well as reduce the stability of the positioning system as a whole. In this regard, an urgent task is the search for opportunities to simplify the requirements for communication lines and the structure of the positioning system based on range-difference location method by reducing the number of receiving 
points involved to a single unmanned aerial vehicle equipped with radio monitoring equipment and ground control and processing point (GCPP).

\section{Range-difference location method}

The purpose of the article is to describe the stages of the approach to determining the coordinates of the pulsed radio signals sources by using a single UAV sensor. Development of a simulation model that allows to identify the main factors affecting the accuracy of pulsed radio signals sources positioning and to estimate its limit values in the framework of the proposed approach. The idea of reducing the number of receiving points in the implementation of rangedifference location method to one UAV sensor and GCPP (Fig. 1), based on the use of features of the modes of some pulsed radio signals sources. It is occurs when a pulse signal with a constant pulse repetition period $(\mathrm{T})$ for a certain period of time $(\Delta \mathrm{t})$ are formed [16]. This feature allows determine the coordinates of these sources without retransmitting the received signal and the organization of a high-precision synchronization system required when using two or more receiving points. The proposed approach includes 10 main stages. They are following below.

Stage 1. Evaluation of initial data.

Stage 2. Search for a pulsed radio signal in a given range of operating frequencies, when a pulsed signal is detected, its parameters are evaluated, namely the pulse repetition period and their duration.

Stage 3. Generates a timestamp grid. The timestamp meshing procedure involves four steps:

- pulse normalization and threshold setting;

- measurement of pulse start time $\left(t_{0}\right)$; period);

- calculation of the timestamp grid $\left(t_{i}=t_{o}+T \cdot i\right.$, where $i$ is the sequence number of the signal

- setting the interrupt timer based on the quantum frequency standard.

Stage 4. Formation of final samples of signal-noise structures. The formation of the reference sample is realized in the sampling block and consists is to record the common-mode and quadrature realization of the detected pulsed radio signal at the first observation point with coordinates $(\mathrm{x}, \mathrm{y}, \mathrm{z})$ with a duration equivalent to the pulse repetition period (T). Further sampling is carried out at each observation point with fixing the coordinates of these points during the entire time of observation of the source $(\Delta \mathrm{t})$.

Stage 5. Estimation of coordinate-informative parameter value. The procedure for estimating the coordinate-informative parameter is to find the time offset of the correlation maximum $\left(t_{i}\right)$, obtained as a result of correlation processing of samples of signal-noise designs ( $\mathrm{Si})$, recorded in the $i^{\text {-th }}$ moments of time, with the reference sample $\left(\mathrm{S}_{0}\right)$, recorded at the first observation point. To do this, the final samples from the sampling unit are fed to the digital correlator. The correlator calculates the modulus of the joint correlation function (JCF) of the final samples. JCF is calculated by discrete convolution of finite samples according to the Equation:

$$
z_{i}=\left|\sum_{s=0}^{2 N-1} S_{0}(s) \cdot S_{i}(n-s)\right|,
$$

where $(2 N-1)$ is the volume of JCF; $s$ is the indexation of the final sample samples; $S_{0}$ and $S_{i}$ are the final samples of signal-noise structures at the first and i-th observation point, respectively.

Time delay values $\left(\tau_{i}\right)$ corresponding to JCF maxima exceeding the threshold are calculated by the Equation:

$$
\tau_{i}=\frac{N-j}{f_{\partial}},
$$

where $j$ is the number of ICF samples exceeding the detection threshold of the PRSS; $f_{\partial}$ is the sample rate. 
Stage 6. Limitation of the duration of the measurement cycle of the coordinate-informative parameter. Accumulation of time delay array is produced during the source control period $(\Delta t)$ :

$$
T \cdot i \leq \Delta t \text {. }
$$

Stage 7. Transmission of observation point coordinates and CIP values to the GCPP. The generated data vector, including the coordinates of observation points and the corresponding CIP value, is transmitted to the GCPP via a communication channel.

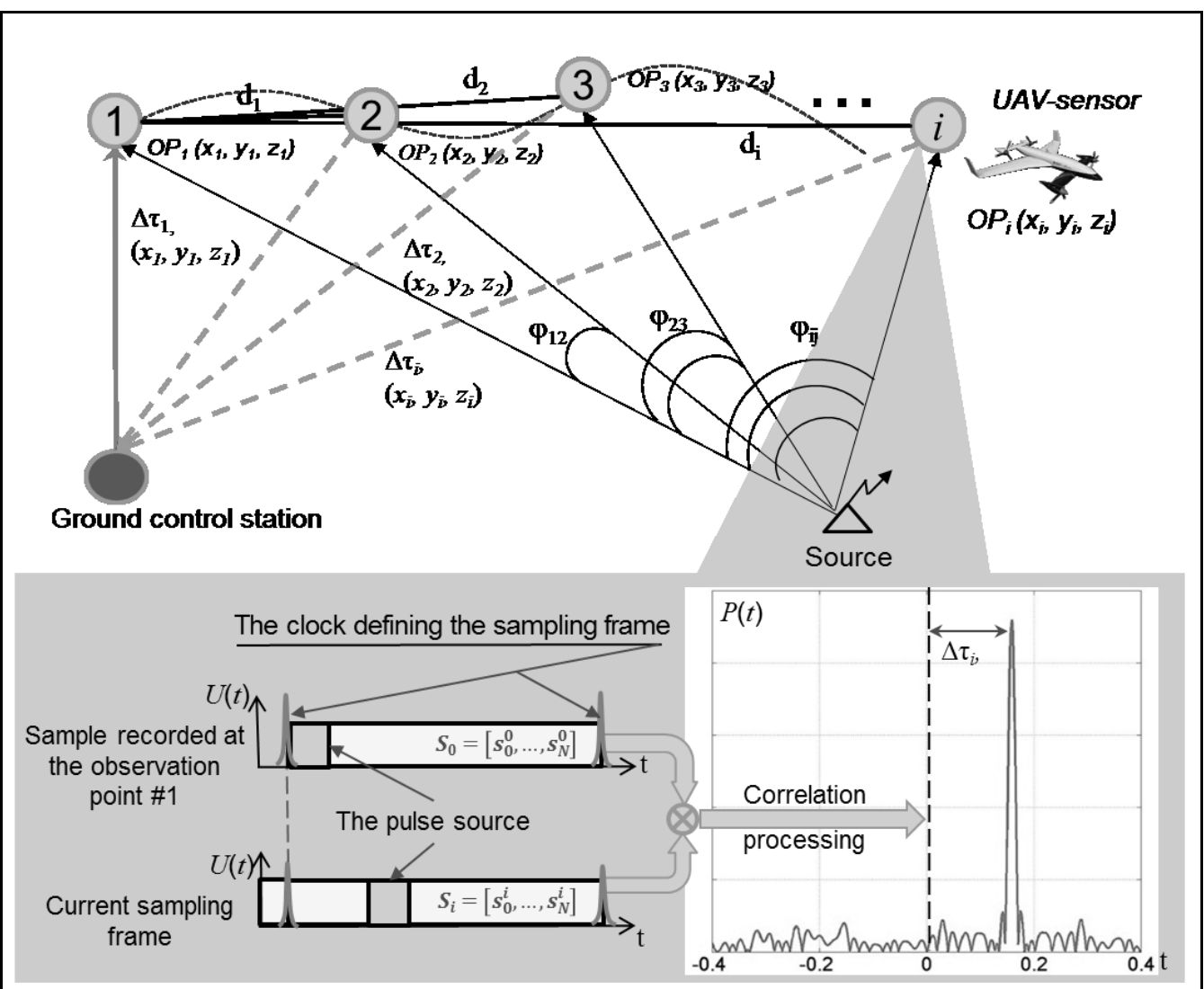

Fig.1. Schematic representation of the implementation of the proposed approach to finding the CIP and determining the coordinates of PRSS

Stage 8. Formation of independent measurements taking into account the geometric factor and the intersection angle.

Stage 9. Calculation of intersection points of position lines and elimination of ambiguity of coordinate estimation. A well-known algorithm is used, with the help of an advanced estimation of the PRSS coordinates on the basis of difference-range measurements [17]. Steps in the disambiguation procedure:

- check for ambiguous solutions;

- calculation of various distances between cross points, selection values and fixations of these points;

- forming points of intersection of two arrays from the common array, according to the condition " which of the fixed points is closer»;

- estimation of the standard deviation of the obtained arrays and selection of the data array with the smallest deviation for further statistical processing.

Stage 10. Statistical processing of intersection points of position lines, estimation of coordinates and their characteristic. 


\section{Experimental part}

In the interests of experimental studies evaluating the potential accuracy of PRSS coordinates determining under the proposed approach, the developed simulation model [18] which allows you to manipulate the dimensions of difference-range location system and the position of the "shiny" points, forming the path of movement of the UAV-sensor, change the energy and time parameters of the source. The received signal has a time delay at the location of the UAV sensor, Doppler frequency shift, additive noise component and several modes formed by a multipath propagation channel:

$$
y(t)=A u(t-\tau) \exp \left(2 \pi j\left[f_{c}+f_{\partial}\right] t\right)+\operatorname{Re}\left[\sum_{i=1}^{M} A\left(\sigma_{i}\right) u\left(t-\tau_{i}\right) \exp \left(2 \pi j\left[\left(f_{c}+f_{\partial i}\right) t-f_{c} \tau_{i}-\varphi_{i}\right]\right)\right]+n(t),
$$

where $A$ is the amplitude of the direct signal; $u(t)$ is the envelope signal; $A\left(\sigma_{i}\right)$ is the amplitude of the reflected signal; $\sigma_{i}$ is the effective surface scattering of the $\mathrm{i}^{\text {th }}{ }^{\text {teflected signal; }} \mathrm{f}_{\partial i}$ is the Doppler shift of the reflected $\mathrm{i}_{-}{ }^{\text {th }}$ signal; $t_{i}$ is the spatial signal delay from $\mathrm{i}^{\text {th }}$ "shiny" point; $\varphi_{i}$ is a random phase shift; $M$ - the number of reflectors on the road; $n(t)$ is additive Gaussian noise.

The accuracy of coordinates determination is estimated by the example of a radio signal generated by a radar station with the following parameters: radiated pulse power of $1 \mathrm{~kW}$, pulse repetition rate of $100 \mu \mathrm{s}$ with a duty cycle of $2 \%$, intra-pulse filling has a carrier frequency of 9.7 $\mathrm{GHz}$ with linear frequency modulation (LFM) and frequency deviation of $5 \mathrm{MHz}$.

\section{Results and discussions}

The result of signal generation based on the proposed analytical model (Equation (4)) in the time and frequency domains are shown in Fig. $2 \mathrm{a}$ and $2 \mathrm{~b}$. The direct signal propagates over the shortest distance and has greater energy characteristics than the reflected ones. When calculating the JCF of the reference sample $\left(S_{0}\right)$ and the signal-noise structures $\left(S_{i}\right)$, the increased energy provides a maximum when convolution of direct signals (Fig. 2c), which allows for the filtering of JCF bursts caused by multipath propagation.

However, there are exceptions to this rule. The direct signal modes formed as a result of reflection from the "shiny" points overcome various distances and are shifted on the time axis when the UAV sensor moves. The interference pattern formed in some cases provides in-phase addition of several modes with a total excess of the direct signal energy.

The described phenomenon leads to significant CIP measurement errors and generates outliers in the resulting sample. CIP measurement outliers are filtered using statistical tools that take into account the possible variation intervals of the measurement result in the current time interval. To calculate the boundaries of the intervals of variation, as well as to plan the experiment, the study of the distribution law of the error in determining the coordinates of the PRSS was performed. Based on the Central limit probability theorem, the hypothesis of the normal distribution of the coordinate determination error is accepted as the null hypothesis $H_{0}$.

Figure $3 a$ shows the dependence of the error variance $R_{M S E(R S)}$ and its average value on the number of experiments in the series $(B)$ at a fixed value of other input factors. The result of Shapiro-Wilk test with significance level $\alpha=0.05$ confirmed the validity of the null hypothesis about the normal error distribution law and provided the possibility of reasonable application of the student's t-test to determine the volume of a representative sample.

Figure 3a shows that the variance of the error $\mathrm{R}_{\mathrm{MSE}(\mathrm{RS})}$ does not undergo significant changes at 100 or more experiments in the series, the result of the t-test showed that the volume of experiments $\mathrm{B}=100$ is sufficient to obtain an unbiased estimate of the average trend of the random variable

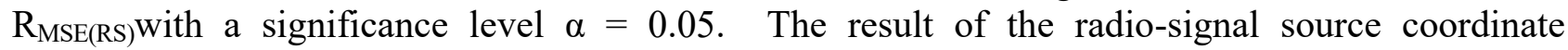
determination depends on several input factors: the geometrical factor of the mutual arrangement of the source and the trajectory of UAV-sensor, the signal-to-noise ratio at the receiving end, the 
number of measurement points coordinate-informative parameters, the positioning accuracy of $\mathrm{UAV}$-sensor, the operating time of the radiation source, etc.

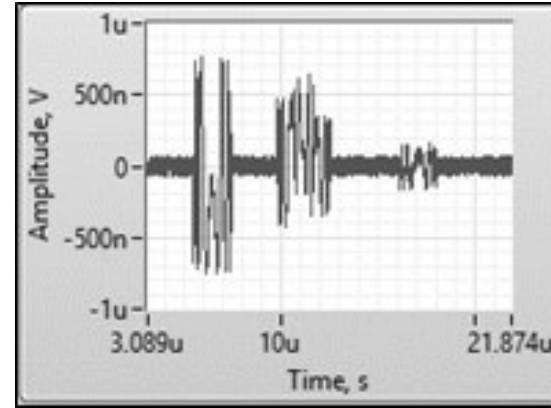

a)

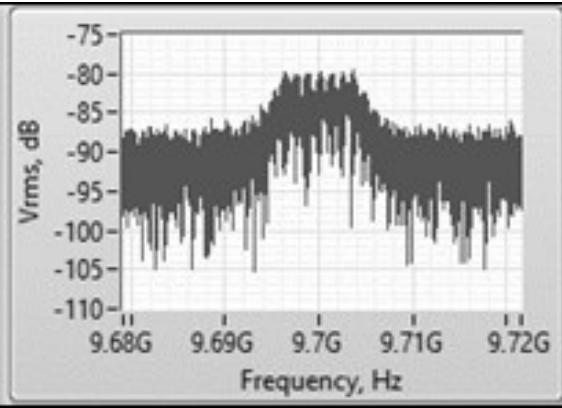

b)

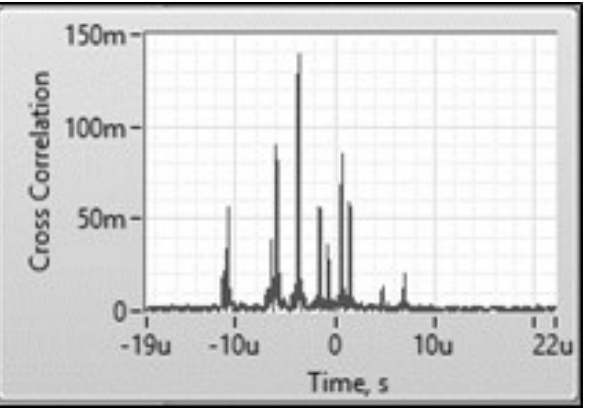

c)

Fig.2. Result of simulation of the PRSS signal at the receiving point:

a) signal in time domain; b) the spectrum of the LFM signal; c) result of JCF calculation

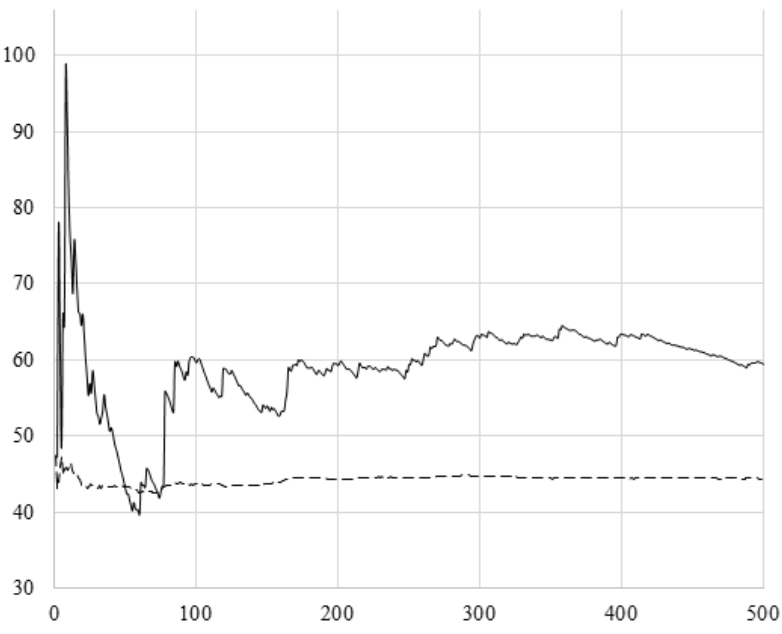

a)

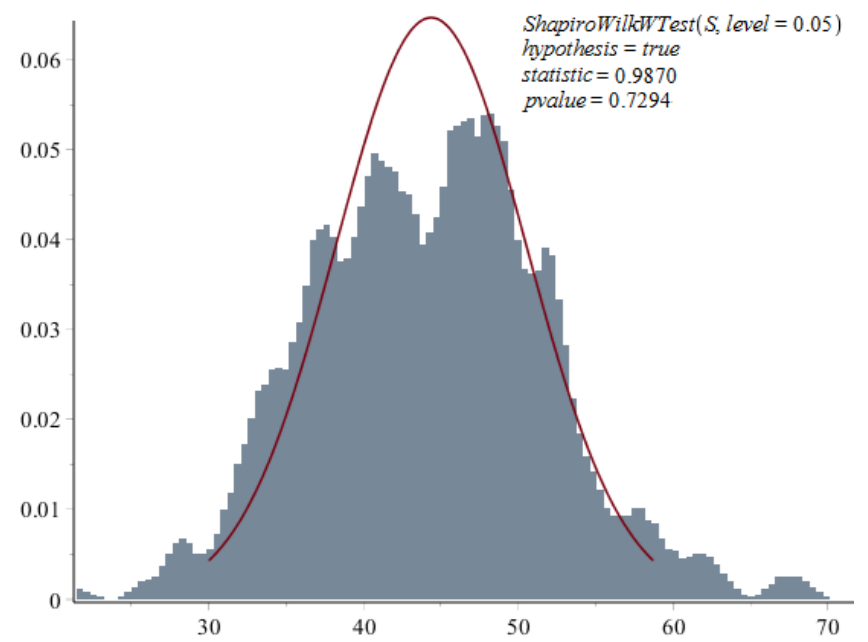

b)

Fig. 3. The result of a statistical study of the distribution law of the coordinate determination error:

a) Variation of deviation and mean error of coordinate determination from the number of experiments; b) Frequency histogram of $\mathrm{R}_{\mathrm{MSE}(\mathrm{RS})}$ error and theoretical probability distribution density

The geometric dilution of precision (GDOP) when using range-difference location method has a significant impact on the value of coordination error $\mathrm{R}_{\mathrm{MSE}(\mathrm{RS})}[17]$. CIP measurements are performed by a mobile UAV sensor, which leads to a change in time of both the GDOP and the signal-to-noise ratio. The GDOP value weighted by SNR's parameters is calculated using the Equation:

$$
D=\frac{\sqrt{\frac{1}{q_{12}\left(2 \sin \frac{\varphi_{12}}{2}\right)^{2}}-\frac{1}{q_{23}\left(2 \sin \frac{\varphi_{23}}{2}\right)^{2}}}}{\sin \gamma}=2 \sqrt{\left(\frac{l_{12}}{q_{12}}+\frac{l_{23}}{q_{23}}\right) l_{13}},
$$

where

$$
l_{12}=\frac{r_{2}\left(r_{2}+\Delta r_{12}\right)}{d_{12}^{2}-\Delta r_{12}^{2}}, \quad l_{23}=\frac{r_{2}\left(r_{2}+\Delta r_{23}\right)}{d_{12}^{2}-\Delta r_{23}^{2}}, \quad l_{13}=\frac{\left(r_{2}+\Delta r_{23}\right)\left(r_{2}+\Delta r_{12}\right)}{d_{13}^{2}-\left(\Delta r_{12}^{2}+\Delta r_{23}\right)^{2}},
$$


where $q_{12}$ and $q_{23}$ are power SNR for measuring mutual delay between measurement points OP1 and OP2, OP2 and OP $3 ; \varphi_{12}, \varphi_{23}$ are angles of sight in the measurement of CIP; $\gamma$ is angle of intersection of position lines; $d_{12}, r_{1}, \ldots \Delta r_{23}$ are geometric dimensions of system elements at measurement points (Fig. 1).

Changing the value of the GDOP and the signal-to-noise ratio is shown in Fig.4. Analysis of the above dependencies allows us to conclude that the geometric factor and the signal-to-noise ratio should be taken into account in the statistical processing of the results of coordination. So at the measuring points (OP $\in[330,390]$ ), signal-to-noise ratio deviation by the superposition of direct and reflected signals, is in the range SNR $\in[7.5 \mathrm{~dB}, 12.5 \mathrm{~dB}]$, which leads to fluctuations of the weighting factor $\mathrm{D}$ twice $\left(\mathrm{D} \in\left[10^{-7}, 2 \cdot 10^{-7}\right]\right)$. Obviously, the use of parameter (D) as a weighting factor in the statistical processing of CIP measurement results will improve the accuracy of determining the coordinates of the PRSS.

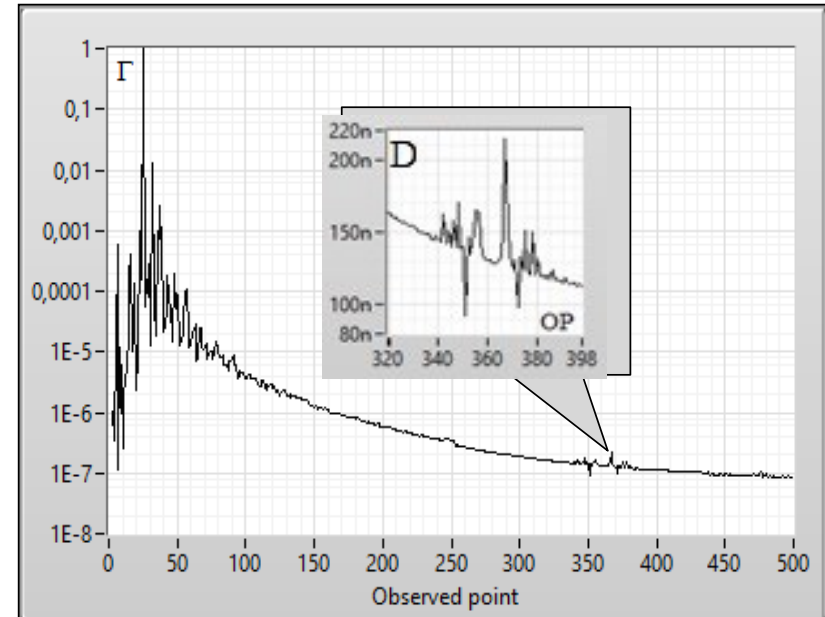

a)

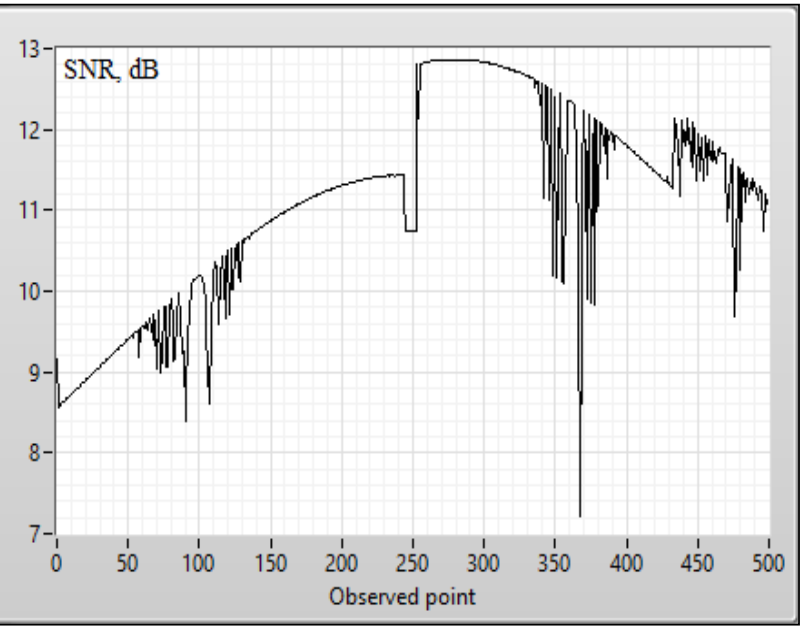

b)

Fig.4. Dynamics of geometric factor and SNR changes during UAV sensor movement

The accuracy of determining the UAV sensor coordinates with other constant values of the input factors also has a significant impact on the error of determining the location of the radio signal source. Statistical estimates of the coordinate measurement error are obtained at the following distance to the source $\mathrm{R}_{\mathrm{UAV}-\mathrm{RS}}=12000 \mathrm{~m}$ and the signal-to-noise ratio $\mathrm{SNR} \geq 15 \mathrm{~dB}$. The analysis of the obtained estimates allows us to conclude that the use of existing positioning systems GPS/GLONASS with $\mathrm{R}_{\mathrm{MSE}(\mathrm{UAV})} \leq 10 \mathrm{~m}$, subject to the application of the proposed approach to coordinate measurement, will ensure the accuracy of determining the coordinates of $\mathrm{R}_{\mathrm{MSE}(\mathrm{RS})} \leq 40 \mathrm{~m}$.

Reducing the distance between the source and the UAV sensor $\left(R_{U A V-R S}\right)$ leads to reduction both the absolute value of the coordinate measurement error and the relative error expressed as a percentage of the distance to the source. In order to determine the conditions and boundaries of the application of the proposed approach, the dependence of the coordinate measurement error on the number of CIP measurement points on the UAV sensor route at different values of the signal-tonoise ratio at the receiving point is studied (Fig.5).

The analysis of the above dependences allows us to conclude that at the level of the received signal SNR $<3 \mathrm{~dB}$ there is a sharp increase in the average error of determining the coordinates of the source. To minimize the error $\left(\mathrm{R}_{\mathrm{MSE}(\mathrm{RS})}\right)$, you should take a sample of the measurement results of the maximum volume CIP ( $\rightarrow$ max). The peculiarity of the proposed approach to determining the location of PRSS is the ability to achieve the required accuracy of the coordination by controlling the measurement time of CIP.

Figure 6 shows the dynamics of reducing the error $\mathrm{R}_{\mathrm{MSE}(\mathrm{RS})}$ with increasing flight time UAV sensor $\Delta t$ for different accuracy of calculating the own coordinates $R_{M S E(U A V)}$. 


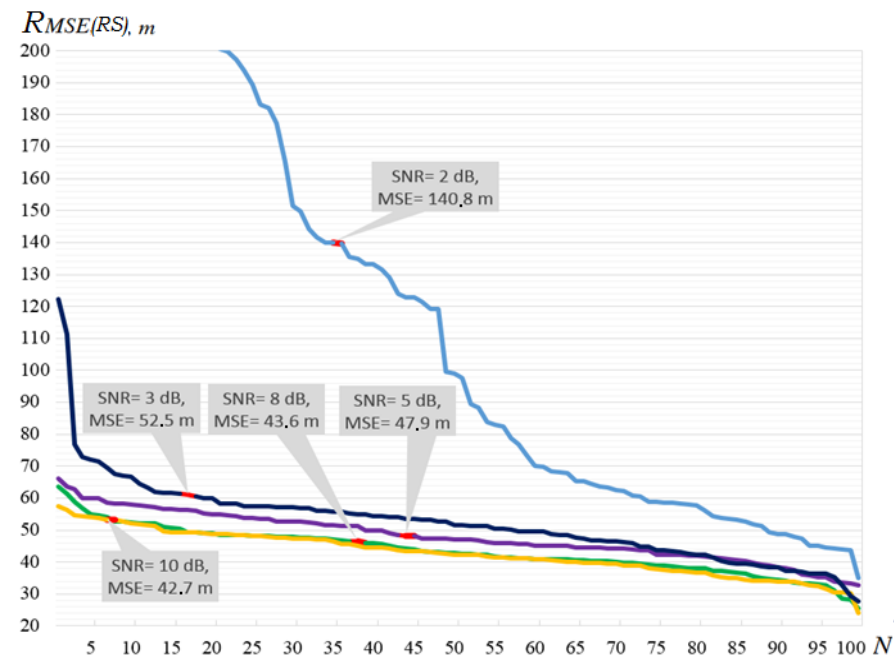

Fig.5. Dependence of the coordinate determination error (MSE) on the number of measurement points on the route at different average signal-to-noise ratio (SNR)

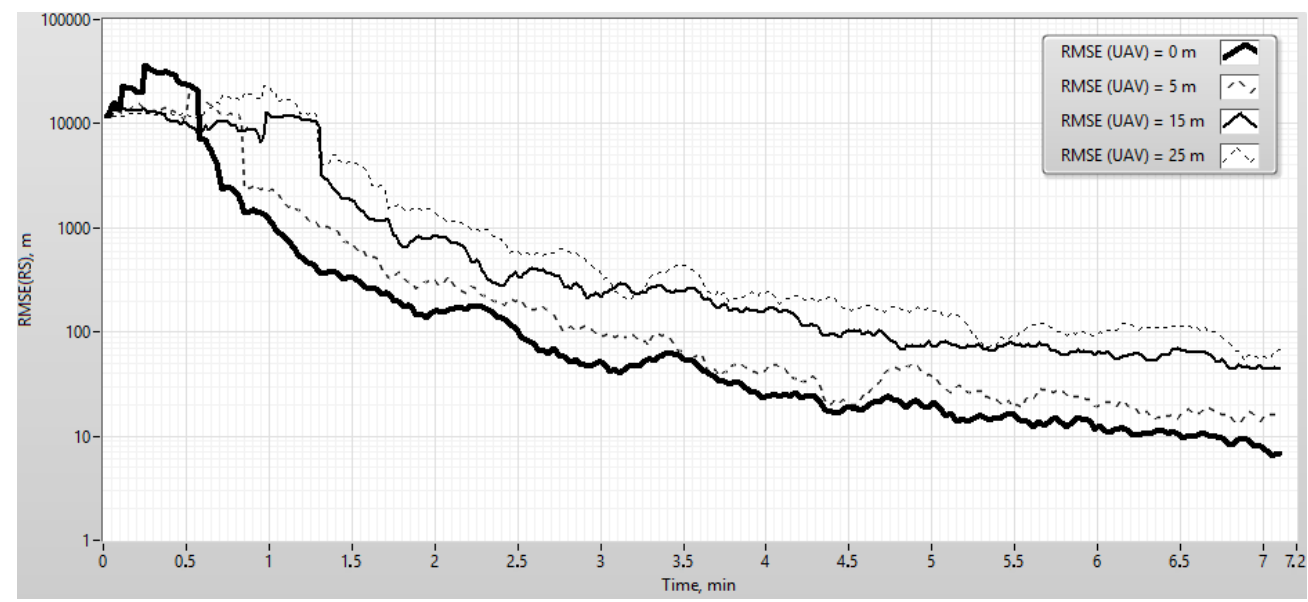

Fig.6. Error in determining the coordinates of $\mathrm{R}_{\mathrm{MSE}(\mathrm{RS})}$ for different accuracy in calculations the own coordinates of the UAV sensor.

Application of global positioning system GPS / GLONASS with $\mathrm{R}_{\mathrm{MSE}(\mathrm{UAV})} \leq 10 \mathrm{~m}$ will allow to achieve accuracy of determination of coordinates $\mathrm{R}_{\mathrm{MSE}(\mathrm{RS})} \leq 110 \mathrm{~m}$ during time of measurement $\Delta t \geq$ 3. To achieve the accuracy of determining the coordinates $\mathrm{R}_{\mathrm{MSE}(\mathrm{RS})} \approx 40 \mathrm{~m}$ the required flight duration of the UAV-sensor to accumulate samples of CIP will be about 5 minutes. These dependences are obtained at a signal-to-noise ratio $\mathrm{SNR} \approx 5 \mathrm{~dB}$ at a distance of $\mathrm{R}_{\mathrm{UAV}-\mathrm{RS}} \approx 12 \mathrm{~km}$ in the presence of three "shiny" points with the reflected signal energy at the receiving point of 30 $70 \%$ of the direct signal power.

\section{Conclusion}

An approach to determining the coordinates of pulsed radio signals sources is based on the range-difference location method, which allows to measure CIP without retransmitting the signal using a single UAV sensor unlike the known methods; it significantly reduces the requirements for communication channels and increases the survivability of the system. The result is achieved through the development of scientific and methodological apparatus that allows using the operation mode features of the pulsed radio signals source, namely the formation of a pulsed signal with a constant pulse repetition period for a certain period of time. 
The lack of errors associated with universal time system and the difference in the amplitudefrequency characteristics of the receiving paths in systems with several receiving points allows to achieve significantly better results in the accuracy of the coordinate determining. It is advisable to use a weighting factor that allows taking into account the GDOP and the signal-to-noise ratio at each measurement point on the UAV sensor route when statistically processing the results of CIP measurements. The required accuracy of coordinate determination can be achieved by changing the CIP sample accumulation time up to $15-20$ meters for the modern technological level of the UAV sensor onboard equipment depending on the tasks to be solved.

\section{REFERENCES}

1 Li C., Weihua Z. Hybrid tdoa/aoa mobile user location for wideband CDMA cellular systems. IEEE Trans. Wireless Commun. 2002, Vol. 1, No. 3, pp. 439 - 447.

2 Dempster A.G. Dilution of Precision in Angle-of-Arrival Positioning Systems. Electron. Lett. 2006, Vol. 42, No.5, pp. 291 - 292.

3 Kułakowski P., Vales-Alonso J., Egea-López E., Ludwin W., García-Haro J. Angle-of-arrival localization based on antenna arrays for wireless sensor networks. COMPUT ELECTR ENG. 2010, Vol. 36, No. 6, pp. $1181-1186$.

4 Xu J., Ma M., Choi L.L. AOA Cooperative Position Localization. Proceeding of the IEEE Global Telecommunications Conference. 2008, pp. 3751- 3755.

5 Du H.J., P.Y. Lee. Technical Memorandum, DRDC Ottawa TM 2004-256, 2004, 24 p.

6 So H.C., Hui S.P. Constrained location algorithm using TDOA measurements. IEICE T FUND ELECTR. 2003, Vol. E86-A, No. 12, pp. 3291- 3293.

7 So H.C., Ching P.C., Chan Y.T. A new algorithm for explicit adaptation of time delay. IEEE T SIGNAL PROCES. 1994, Vol. 42, No.7, pp. 1816 - 1820.

8 Featherstone W., Strangeways H. Mobile transmitter AOA estimation under multipath conditions using an MLE based super resolution algorithm and comparison with weighted spectrum methods. Proceeding of the IEE Colloquium Novel Methods of Location and Tracking of Cellular Mobiles and their System. 1999, pp. 4 - 4, doi: 10.1049/ic:19990241.

9 Pages-Zamora A., Vidal J., Brooks D.H. Closed-form solution for positioning based on angle of arrival measurements. Proceeding of the 13th IEEE International Symposium on Personal, Indoor and Mobile Radio Communications. 2002, Vol. 4, pp. 1522 - 1526.

10 Ho K., Chan Y. Solution and performance analysis of geolocation by TDOA. IEEE T AERO ELEC SYS. 1993, Vol. 29, No. 4, pp. $1311-1322$.

11 Ho K.C., Chan Y.T. Geolocation of a known altitude object from TDOA and FDOA measurements. IEEE T AERO ELEC SYS. 1997, Vol. 33, No.3, pp. 770 - 783.

12 Hashemi-Sakhtsari A., Dogancay K. Recursive Least Squares Solution to Source Tracking using Time Difference of Arrival. Proceeding of the IEEE International Conference on Acoustics, Speech and Signal Processing. 2004, Vol. 2, pp. 385 - 388.

13 Lee S.C., Lee W.R., You K.H. TDoA Based UAV Localization Using Dual-EKF Algorithm. In: Ślęzak D., Kim T., Stoica A., Kang BH. (eds). Control and Automation. 2009, Vol. 65, pp. 47 - 54.

14 Venkatraman S., Caffery J.J. Hybrid TOA/AOA Techniques for Mobile Location in Non-LineofSight Environments. Proceeding of the IEEE Conference Wireless Communications and Networking, 2004, Vol. 1, pp. $274-278$.

15 Cheung K.W., So H.C., Ma W.K., Chan Y.T. A constrained least squares approach to mobile positioning: algorithms and optimality. EURASIP J ADV SIG PR. 2006, Vol. 1, pp. 1 - 23.

16 Baldychev M.T., Pivkin I.G., Gaychuk Yu.N., Pechurin V.V., Laptev I.V. A method for determining the location of sources of pulsed radio signals. Russian Federation Patent No. 2695321, 2019, $10 \mathrm{p}$.

17 Demichev I.V., Yevgeniev D.N., Shaidulin Z.F. Estimation of coordinates of object in view of influence of the geometrical factor. Journal Science Intensive Technologies. 2010, No. 9, pp. 44 - 50.

18 Pivkin I.G., Pechurin V.V., Baldychev M.T., et al. Model of a one - position system for determining the location of radio emission sources. Russian Federation Certificate of State Registration of a Computer Program No.2019613634, 2019, 1 p. 